\title{
Preparation and Characterization of Porous Polymethylmethacrylate Film Showing Optical Reflectivity
}

\begin{abstract}
Jihoon Kim ${ }^{\dagger}$
This paper describes a method for the preparation of porous polymethylmethacrylate showing optical reflectivity from the porous silicon template. A porous polymethylmethacrylate showing optical reflectivity was prepared by replicating porous silicon template which was obtained by applying a computer-generated periodic square current density and resulted in a mirror with high reflectivity in a specific narrow spectral region. A porous polymethylmethacrylate showing an excellent reflectivity was successfully obtained by dissolving the Porous silicon template from the porous polymethylmethacrylate composite film. A porous polymethylmethacrylate exhibited a sharp reflection resonance in the reflectivity spectrum. Surface image of the porous polymethylmethacrylate indicated that the surface of the porous polymethylmethacrylate film had a porous structure. These porous polymethylmethacrylate films in aqueous solutions were stable for several days without any degradation.
\end{abstract}

Key words: Reflection, Porous Polymer, Porous Silicon, Polymethylmethacrylate

\section{Introduction}

Discovering of new optical materials based on novel physical and chemical properties represents one of the most interesting challenges in modern materials science. Especially, flexible optical polymethylmethacrylate thin films having porosity are particularly attractive $\mathrm{e}^{[1]}$. Recently, the addition of functionalities to polymer materials has led to markedly enhanced control of a wide range of technically important material properties from optoelectronic to mechanical properties ${ }^{[2]}$. The optical polymer thin films having porosity have advantages of flexible possibilities. However, studies on the porous polymethylmethacrylate exhibiting the reflection optical properties are very limited.

Since the discovery of porous silicon by Cahnam $^{[3]}$, porous silicon has been investigated for various applications, such as biological and chemical sensors, medical diagnostics, optical filters, micro chemical reactors, and fuel cells. Optical devices based on porous silicon have been brought to the attention of scientists ${ }^{[4-15]}$. Porous silicon has advantages for building optical structures as well as very high surface area with various surface chemistry and unusual optical properties. The

Nanolamda, National Nanofab Center, Daejon 305-806, Korea

${ }^{\dagger}$ Corresponding author: jeuse@hanmail.net

(Received: February 7, 2013, Revised : June 20, 2013

Accepted : June 24, 2013) porosity and pore size of porous silicon can be controlled by modulating the applied current densities ${ }^{[16-20]}$. Porous polymer materials showing optical properties could be an alternative, since porous silicon has the limitation for chemical and mechanical stability for many applications. This provides the means to construct complex porous structures of polymer thin film that are compatible with harsh environments and to improve chemical and mechanical stability.

\section{Experimental Section}

\subsection{Preparation of Porous Silicon}

The porous silicon samples were prepared by electrochemically etching heavily-doped $\mathrm{p}^{++}$-type silicon wafers (boron doped, polished on the $<100>$ face, resistivity of $0.8-1.2 \mathrm{~m} \Omega \cdot \mathrm{cm}$, Siltronix, Inc.). The etching solution consisted of a $3: 1$ volume mixture of $48 \%$ aqueous hydrofluoric acid (ACS reagent, Aldrich Chemicals) and absolute ethanol (ACS reagent, Aldrich Chemicals). A galvanostatic etch was carried out in a Teflon cell by applying 20 cycles of a two-electrode configuration with a Pt mesh electrode. DBR porous silicon was prepared by using periodic square wave currents between $5 \mathrm{~mA} \cdot \mathrm{cm}^{-2}$ for $75 \mathrm{~s}$ and $50 \mathrm{~mA} \cdot \mathrm{cm}^{-2}$ for $2 \mathrm{~s}$. The anodization current was supplied by a Keithley 2420 high-precision constant current source controlled by a computer to allow the formation of porous silicon 
multilayers. To prevent the photogeneration of carriers, we performed the anodization in the dark. All the samples were then rinsed several times with ethanol and dried were under an Ar atmosphere prior to use. Freestanding porous silicon films were obtained from the silicon substrate by applying as electropolishing current of $360 \mathrm{~mA} \cdot \mathrm{cm}^{-2}$ for $1 \mathrm{~min}$ in an ethanoic $37.5 \%$ aqueous HF solution and of $24 \mathrm{~mA} \cdot \mathrm{cm}^{-2}$ for $2 \mathrm{~min}$ in an ethanoic $3.3 \%$ aqueous HF solution.

\subsection{Preparation of Porous Polymethylmethacrylate} Thin Film

Prepared free-standing porous silicon films were thermally oxidized in a furnace at $300^{\circ} \mathrm{C}$ for $3 \mathrm{hrs}$. For the replicating solution, $3 \mathrm{~g}$ of polymethylmethacrylate (Aldrich, $\mathrm{Mw}=120,000$ ) was dissolved in $20 \mathrm{~mL}$ of toluene (Fisher Scientific). The resulting mixtures were cast onto porous $\mathrm{SiO}_{2}$ film and the samples were annealed in an oven at $95^{\circ} \mathrm{C}$ for $1 \mathrm{hr}$. Then, the oxidized porous silicon was removed in $0.1 \mathrm{M}$ aqueous $\mathrm{NaOH}$ for $3 \mathrm{hrs}$.

\subsection{Instrumentation and Data Acquisition}

The optical reflectivity spectra were measured using a tungsten-halogen lamp and an Ocean Optics S2000 CCD spectrometer fitted with a fiber optic input. The reflected light collected at the end of the fiber optic was positioned at the focal plane of an optical microscope. FT-IR spectra were acquired with a Nicolet model 5700 FT-IR instrument in the diffuse reflectance mode (Spectra-Tech diffuse reflectance attachment). The FT-IR sample compartment was purged with nitrogen before each acquisition. The morphology of sample was observed with FE-SEM (S-4700, Hitachi)

\section{Results and Discussion}

Schematic diagram for the preparation of porous polymethylmethacrylate thin films are shown in Figure 1. A porous polymethylmethacrylate showing optical reflectivity was prepared by replicating porous silicon template which was obtained by applying a computergenerated periodic square current density. Porous silicon exhibits a high reflectivity band with a Bragg wavelength $\lambda_{\text {Bragg }}$ that depends on the thicknesses of the layers $\left(d_{1}, d_{2}\right)$ and the corresponding refractive indices $\left(n_{1}\right.$, $n_{2}$ ). The $m$ th order of the Bragg peak is given by

$$
m \lambda_{\text {Bragg }}=2\left(d_{1} n_{1}+d_{2} n_{2}\right) .
$$

Typical etch parameters for the porous silicon structure involve a periodic square wave current between low and high current densities. For the fabrication of porous silicon, an applied current densities used between 5 and $50 \mathrm{~mA} \cdot \mathrm{cm}^{-2}$. The etching times for a $\lambda /$ 4 layer of a Bragg structure were typically $75 \mathrm{~s}$ for a low current and $2 \mathrm{~s}$ for a high current. The reflection peak shown in Fig. 2 in the reflection spectrum showed a narrow full width at half maximum ca. $22 \mathrm{~nm}$ at $610 \mathrm{~nm}$. The resulting porous silicon films were lifted off from the silicon wafer to obtain porous silicon films

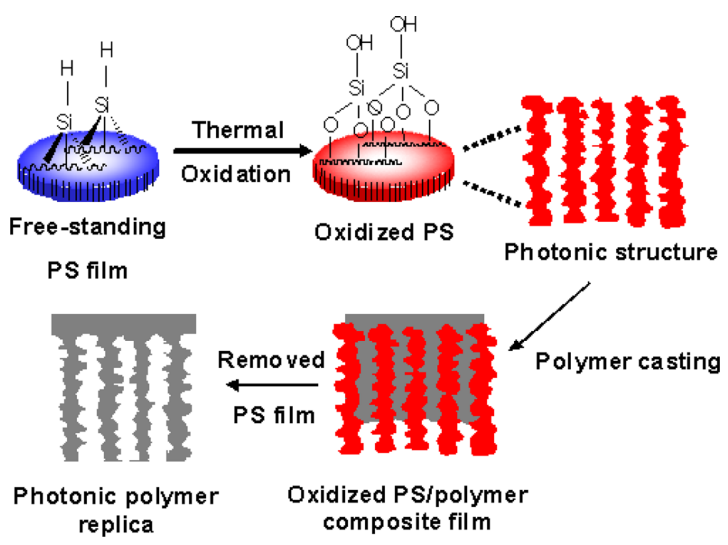

Fig. 1. Schematic diagram for the preparation of porous polymethylmethacrylate thin film.

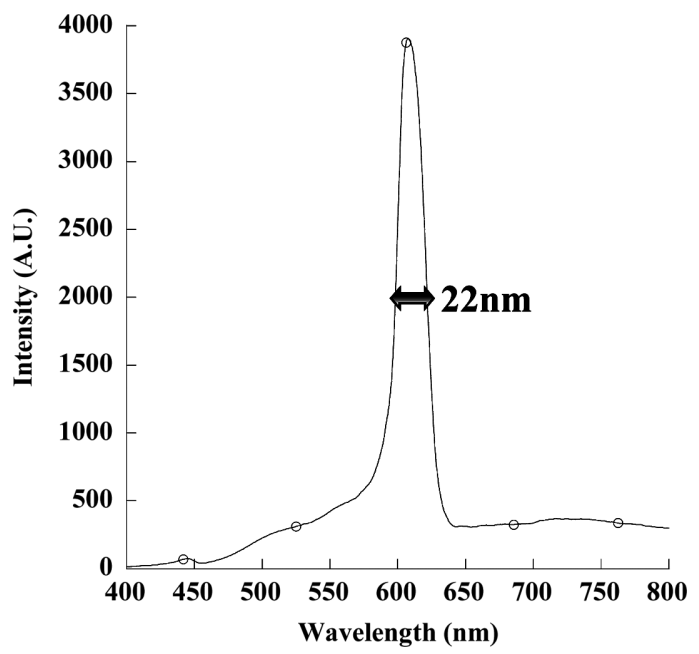

Fig. 2. Optical reflectivity spectrum of porous silicon films. 
by applying of electropolishing current in a solution of HF and ethanol. The reflection peak of porous silicon film was observed at $610 \mathrm{~nm}$. Oxidation of porous silicon film was thermally carried out in a furnace at $300^{\circ} \mathrm{C}$ for $4 \mathrm{hrs}$. The reflection peak for the oxidized porous silicon film displayed reflectivity at $570 \mathrm{~nm}$, which were shifted to shorter wavelengths. This is due to a decrease of the average refractive index from that for silicon to that for silicon dioxide.

After the thermal oxidation of the porous silicon film, the presence of silicon oxide was determined by using FT-IR measurement, as shown in Figure 3. The FT-IR spectrum of a fresh porous silicon film displayed vibrational bands in the fingerprint region of the spectrum. $\mathrm{U}_{(\mathrm{Si}-\mathrm{H})}$ and $\delta_{(\mathrm{Si}-\mathrm{H})}$ vibrations associated with surface $\mathrm{Si}-$ $\mathrm{H}$ species were also apparent at 2117 and $941 \mathrm{~cm}^{-1}$, respectively. Thermal oxidation of the porous silicon layer resulted significant loss of intensity of the $v$ ( $\mathrm{Si}-$ $\mathrm{H})$ modes in the infrared spectrum at $2150 \mathrm{~cm}^{1}$, but vibrational bands due to oxygen-back-bonded silicon hydride, $\mathrm{v}(\mathrm{OSi}-\mathrm{H})$ and $\delta(\mathrm{OSi}-\mathrm{H})$ modes, grew at 2300 and $850 \mathrm{~cm}^{1}$, respectively. Multiple silicon oxide species, $\mathrm{Si}-\mathrm{O}-\mathrm{Si}$, displayed a strong, very broad absorption band between 1000 and $1250 \mathrm{~cm}^{1}$.

The surface morphology of porous silicon was obtained with cold FE-SEM and shown in Figure 4. FESEM image of porous silicon surface indicated that the porous silicon exhibited very stable and even surface. FE-SEM image of porous silicon indicates that the prepared porous silicon has cylindrical mesopores with the pore size of few nanometers and the depth of few microns.

To obtain the porous polymer thin films, the

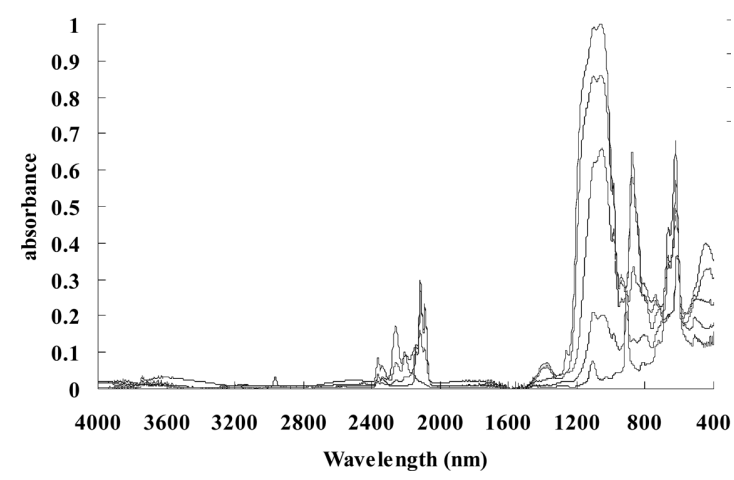

Fig. 3. FT-IR spectra of oxidized porous silicon films. polymethylmethacrylate solution was cast on the top surface of the oxidized porous silicon film. After drying in an room temperature, the resulting composite film was annealed in an oven at $95^{\circ} \mathrm{C}$ to fill the pores of porous silicon with the polymethylmethacrylate. Since top side of the oxidized porous silicon films were coated with the polymethylmethacrylate, the silicon oxide of oxidized porous silicon matrix can be easily removed from the composite films in a dilute aqueous sodium hydroxide solution. X-ray diffraction pattern of the porous polymethylmethacrylate thin film indicates that the oxidized porous silicon template was completely removed from the composite films and that no crystalline silicon was remaining. The surface morphology of porous polymethylmethacrylate thin film was obtained with cold FE-SEM and shown in Figure 5. FE-SEM image of surface of porous polymethylmethacrylate thin film indicated that the prepared porous polymethylmethacrylate thin film has cylindrical mesopores with

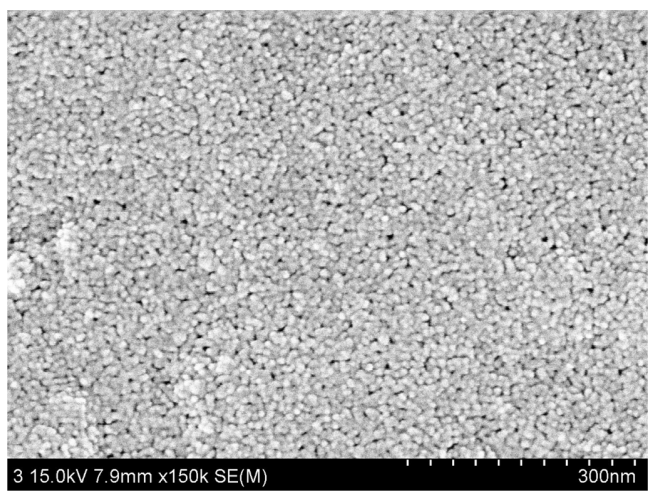

Fig. 4. Surface SEM image of porous silicon

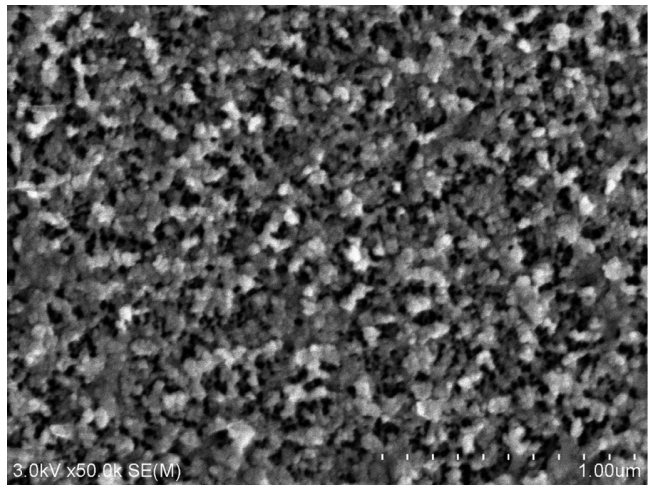

Fig. 5. Surface SEM image of porous polymethylmethacrylate thin film. 


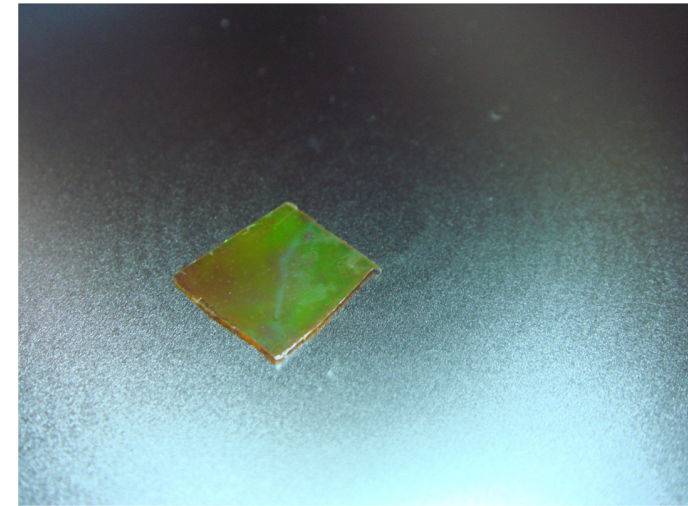

Fig. 6. Photo image of porous polymethylmethacrylate thin film.

the pore size of $100 \mathrm{~nm}$ and the depth of few microns.

After removal of the oxidized porous silicon from the composite film, the porous polymethylmethacrylate thin film exhibited a reflection peak at $570 \mathrm{~nm}$. Porous polymethylmethacrylate thin film shown in Figure 6 exhibits a green color due to the reflection peak of the porous polymethylmethacrylate. The polymethylmethacrylate replica was also highly flexible and displayed a significantly improved mechanical stability without apparent degradation. Its optical properties were retained upon flexing. This method provides a general means of fabricating the photonic porous polymer thin films. The photonic porous polymer thin films possessed stable spectral features and increased stability to corrosion.

\section{Conclusion}

Porous polymer thin films showing high reflectivity were prepared by casting a polymer solution onto the oxidized porous silicon and then removing silicon dioxide of the oxidized porous silicon template from the polymethylmethacrylate composite film. The porous polymer thin films were robust and flexible. They exhibited excellent reflectivity in the reflection spectrum. The methods based on porous silicon have been provided for the preparation of photonic polymer having porous structures.

\section{References}

[1] A. C. Balazs, T. Emrick, and T. P. Russell, "Nano- particle polymer composites: where two small worlds meet", Science, Vol. 314, pp. 1107-1110, 2006.

[2] E. T. Thostenson, C. Y. Li, and T. W. Chou, "Nanocomposites in context", Compos. Sci. Technol. Vol. 65, pp. 491-516,2005.

[3] L.T. Canham, "Silicon quantum wire array fabrication by electrochemical and chemical dissolution of wafers", J. Appl. Phys. Lett. Vol. 57, pp. 1046-1048, 1990.

[4] H. Sohn, S. Letant, M. J. Sailor, and C. Trogler, "Detection of fluorophosphonate chemical warfare agents by catalytic hydrolysis with a porous silicon interferometer", J. Am. Chem. Soc. Vol. 122, pp. 5399-5400, 2000.

[5] M. W. Hwang and S. D. Cho, "Detection of organic vapors using change of Fabry-Perot fringe pattern of surface functionalized porous silicon", J. Chosun Natural Sci., Vol. 3, pp. 168-173, 2010.

[6] D. H. Jung, "Biosensor based on distributed Bragg reflector photonic crystals for the detection of protein A", J. Chosun Natural Sci., Vol. 3, pp. 33-37, 2010.

[7] S. D. Cho, "Preparation of polystyrene thin films containing Bragg structure and investigation of their photonic characteristics", J. Chosun Natural Sci., Vol. 3, pp. 138-142, 2010.

[8] S. D. Cho, "Detection of nitroaromatic compounds with functionalized porous silicon using quenching photoluminescence", J. Chosun Natural Sci., Vol. 3, pp. 202-205, 2010.

[9] Y. D. Koh, "Analysis on oxidation of porous silica obtained from thermal oxidation of porous silicon", J. Chosun Natural Sci., Vol. 3, pp. 153-156, 2010.

[10] J. M. Han, "Photoluminescence of porous silicon according to various etching times and various applied current densities", J. Chosun Natural Sci., Vol. 3, No. 3, pp. 148-152, 2010.

[11] S. H. Jang, "Study on thickness of porous silicon layer according to the various anodization times", J. Chosun Natural Sci., Vol. 3, pp. 206-209, 2010.

[12] K. S. Jung, "Fabrication and characterization of DBR porous silicon chip for the detection of chemical nerve agents", J. Chosun Natural Sci., Vol. 3, pp. 237-240, 2010.

[13] Y. C. Koh, "1-D photonic crystals based on Bragg structure for sensing and drug delivery applications", J. Chosun Natural Sci., Vol. 4, pp. 11-14, 2011.

[14] S. G. Kim, "Optical characterization of smart dust based on photonic crystals and its sensing applica- 
tions", J. Chosun Natural Sci., Vol. 4, pp. 7-10, 2011.

[15] S. H. Jang, "Chemical and physical properties of porous silicon", J. Chosun Natural Sci., Vol. 4, pp. 1-6, 2011.

[16] B.-Y. Lee, M. Hwang, H. Cho, H.-C. Kim, and S. Jang, "Characterization and surface-derivatization of porous silicon", J. Chosun Natural Sci., Vol. 4, pp. 182-186, 2011

[17] B.-Y. Lee, M. Hwang, H. Cho, H.-C. Kim, and S. Jang, "Chemical and physical properties of porous silicon", J. Chosun Natural Sci., Vol. 4, pp. 187-191, 2011.
[18] B.-Y. Lee, M. Hwang, H. Cho, H.-C. Kim, and S. Cho, "Multiple-bit encodings of bragg photonicstructures by using consecutive etch with various square wave currents", J. Chosun Natural Sci., Vol. 4, pp. 192-196, 2011.

[19] B. Kim, "Investigation of relationship between reflection resonance and applied current density in bragg photonic crystal", J. Chosun Natural Sci., Vol. 5, pp. 27-31, 2011.

[20] S. Jang, "Investigation of the changes of FabryPerot fringe patterns in porous silicon during etching process", J. Chosun Natural Sci., Vol. 5, pp. 1317, 2011. 